\title{
Simulation of Human Eye Optical System Properties and Depth of Field Variation
}

\author{
Zilong Wang and Shuangjiu Xiao
}

\begin{abstract}
Human eyes are not perfect optical systems, most of them have refractive errors, the simulation of which is a plus to the realistic vision rendering. This paper presents the simulation of the properties of human eye optical system with an abstracted human eye optical model, including myopia, hyperopia and astigmatism. A novel and effective ray-tracing-based method is brought out in this paper, which calculates the direction of rays refracted by the eye without modeling the surface of the crystalline lens. In order to achieve better simulation results, a circinate-patterned sampling method is proposed, which covers most of the radiation angles and has a distribution similar to Gaussian distribution. The variation of depth of field accompanied by the adjustment of the pupil size in reaction to the luminance of the environment is also simulated.
\end{abstract}

Index Terms - Ray tracing, myopia, hyperopia, astigmatism, depth of field, human eye optical system.

\section{INTRODUCTION}

What we see in the eye is different from others - no leaves are exactly the same, so as human eyes. The new era of virtual reality calls for the simulation of a particular individual's vision.

Human eye is not a perfect optical system, refractive errors may exist in most of the human eyes. In this paper, the simulation of human eye optical system properties refers to the simulation of myopia, hyperopia and astigmatism.

Myopia is a common visual defect [1] that parallel light focus in front of the retina [2]. Distant objects are blurry in myopia eyes, while the near objects can still be clear.

Hyperopia is a defect of vision that the eyeball is too short compared with the focal length of the lens [3]. Subjects with hyperopia will have difficulty focusing on near objects.

Astigmatism is another ordinary optical defect, accounting for about $13 \%$ human eye refractive errors [4]. The vision is blurred due to an irregular curvature of the lens [5], which may be one of the causes of astigmatism.

The size of pupil in human eye will change in reaction to the luminance of the environment [6]. When the environment becomes darker, the pupil will enlarge to allow more light to enter the eye. The change of the size of pupil will result in the variation of depth of field [7]. A larger pupil size leads to a smaller depth of field, and a smaller pupil size is corresponding to a larger depth of field.

Taking account of vision defects and depth of field

Manuscript received July 1, 2013; revised September 15, 2013.

The authors are with the School of Software, the Shanghai Jiao Tong University, Shanghai, 200240 China (e-mail: zilong@ sjtu.edu.cn, xsjiu99@cs.sjtu.edu.cn). variation, a particular individual's vision be presented in this paper in order to obtain vision realistic effect on the render results of virtual reality.

\section{RELATED WORKS}

\section{A. Human Eye Optical System Simulation}

Vision-realistic rendering is introduced by Barsky et al. in a poster at Eurographics 2002 [8], and the details can be referred to in [9], which presents synthetic images showing the characteristics of a particular individual's optical system. Several depth images are created and blurred according to the input image and corresponding depth map. The final synthetic image is composed by these depth images. The concept is novel in that time, but the approach using image processing may not be realistic enough.

In Wolfgang's approach [10], ray tracing is used to simulate various eye defects. It requires complex surface modeling and the refraction is calculated according to Snell's law. Large amount of computation will be needed in this approach.

\section{B. Depth of Field}

In the domain of depth of field, Potmesil and Chakravarty first proposed an optical lens model to simulate depth of field [11]. An efficient way to render depth of field effect using ray tracing is introduced in [12], which gets rid of the computation of every refraction according to Snell's law, and the directions of the refracted rays are derived from focal distance of the lens.

\section{Pupil Size and Luminance}

About the relationship between the pupil size and the luminance of the environment, several researchers proposed their formulas, including Blackie and Howland [13], Winn et al. [14], Stanley and Davies [15] and much more, taking parameters like the luminance of environment, the age, field diameter into consideration.

\section{Human Eye Optical System Simulation}

In this section, the details of the ray-tracing-based method to calculate the direction of rays refracted by the eye are presented with figures showing optical paths of myopia, hyperopia and astigmatism in the abstracted human eye optical model.

\section{A. Abstracted Human Eye Optical Model}

Human eye is a complicated optical system, simulating the entire optical system will consume large amounts of 
computing resources. Considering ray tracing is also time-consuming, the optical model of human eye should be as simple as possible.

In this paper, the optical model of human eye is abstracted to a pupil, a convex lens and a retina, as shown in Fig. 1.

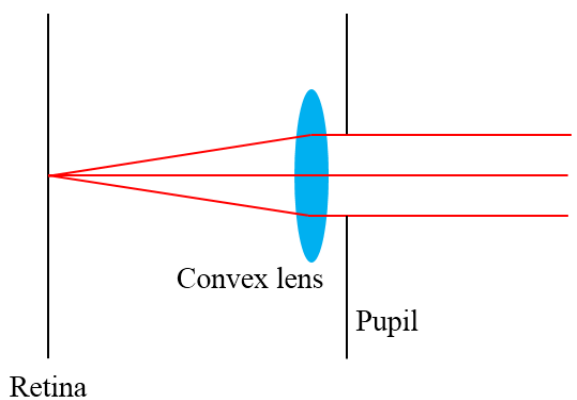

Fig. 1. Optical model of human eye.

The pupil functions as the aperture of camera, controlling how much light can enter the optical model.

The retina is where the rendered image is formed.

The convex lens represents everything in human eye that will bend the light. It refracts the light passed through the pupil.

\section{B. Simulation of Myopia}

In the simulation of myopia, the key process is the calculation of the path of each ray.

The main cause of myopia is that the light entered the eye focus in front of the retina, the optical path is shown in Fig. 2.

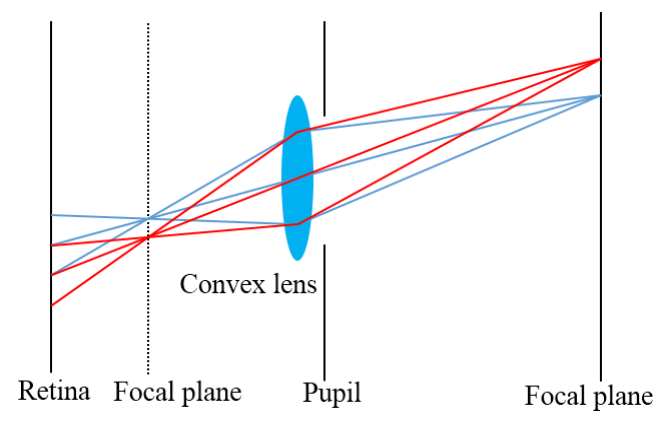

Fig. 2. The optical path of myopia.

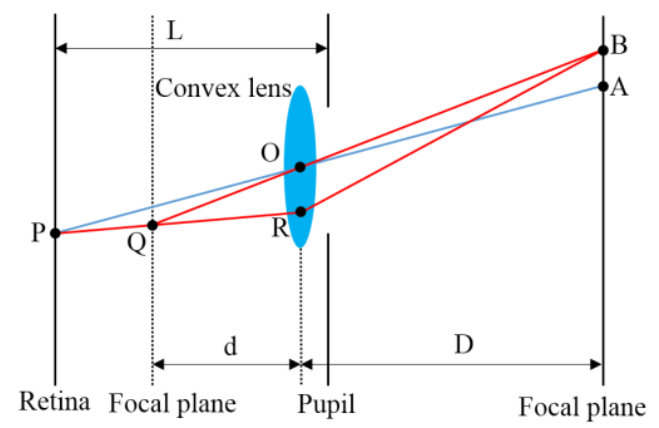

Fig. 3. The optical path of myopia with less rays.

In Fig. 2, the focal plane on the left is the plane where the light focus in the eye, and the focal plane on the right is outside of the eye.

For each single ray, tracing every refraction ray requires the curvature of the convex lens, the calculation of contact points, and the direction of the ray after it pass through the contact point. The simulation process obviously consumes tremendous computing resources.
We consider a simplified method to achieve the same goal, but is much more effective.

For a cleaner view and better illustration, let's focus on some of the rays in Fig. 2, as shown in Fig. 3.

In Fig. 3, point $\mathrm{O}$ is the center of the convex lens. Point $\mathrm{Q}$ is the intersect point of $\overrightarrow{P R}$ and the focal plane on the left. $L$ is the length of the eye. $D$ is the focal distance of the convex lens and $d$ is the focal length of the convex lens.

Please note that the rays in Fig. 3 are exactly the same ray in Fig. 2.

For each point $\mathrm{P}$ in retina, consider ray $\overrightarrow{P O}$ : after it passes through the convex lens, its direction is very easy to calculate, because the rays pass through the center of convex lens will not change the direction.

$$
\overrightarrow{O A}=\frac{D}{L} \overrightarrow{P O}
$$

For each point $\mathrm{R}$ in the convex lens, consider ray $\overrightarrow{P R}$ : after refracted by the convex lens, its direction becomes $\overrightarrow{R B}$, where point B's coordinate is unknown for now. Let's connect point $\mathrm{Q}$ and point $\mathrm{O}$, and extend it until it intersect with the focal plane on the right, assume that the intersect point is point $\mathrm{C}$. It is known that every ray emitted from a point in the focal plane will focus on the same point in the other focal plane after the ray is refracted by a convex lens. So the ray $\overrightarrow{O C}$ and the ray $\overrightarrow{R B}$ will focus on the same point. In other words, point $B$ and point $C$ is the same point. Now the direction of $\overrightarrow{R B}$ can be calculated.

$$
\overrightarrow{R B}=\overrightarrow{R O}+\overrightarrow{O B}
$$

where

$$
\begin{aligned}
\overrightarrow{O B} & =\frac{D}{d} \overrightarrow{Q O} \\
\overrightarrow{Q O} & =\overrightarrow{Q R}+\overrightarrow{R O} \\
\overrightarrow{Q R} & =\frac{d}{L} \overrightarrow{P R}
\end{aligned}
$$

To sum up,

$$
\overrightarrow{R B}=\frac{D+d}{d} \overrightarrow{R O}+\frac{D}{L} \overrightarrow{P R}
$$

Now the direction of every single ray that will eventually hit the retina can be calculated, thus the simulation of myopia is done.

\section{Simulation of Hyperopia}

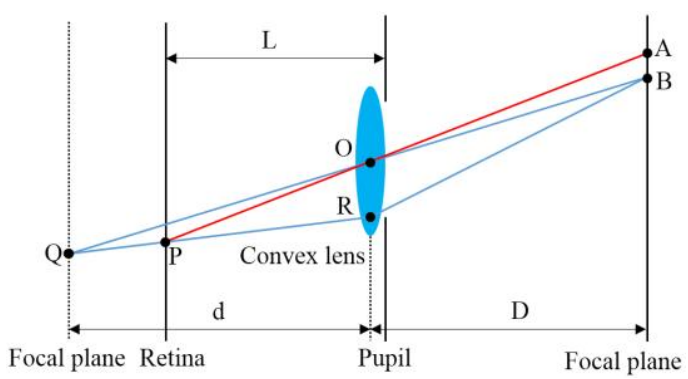

Fig. 4. The optical path of hyperopia with less rays. 
The difference between hyperopia and myopia is that the focal length of the convex lens in hyperopia is larger than the length of the eye.

The optical path can be simplified as the simulation method of myopia, as shown in Fig. 4.

The calculation of the direction of each ray also resembles the one in myopia simulation.

\section{Simulation of Astigmatism}

The main cause of astigmatism is that the curvatures of different meridian of the lens in the eye are not consistent.

There are different types of astigmatism, but in this paper, only the regular astigmatism, which the principal meridians are perpendicular, is included.

The optical path of astigmatism is shown in Fig. 5.

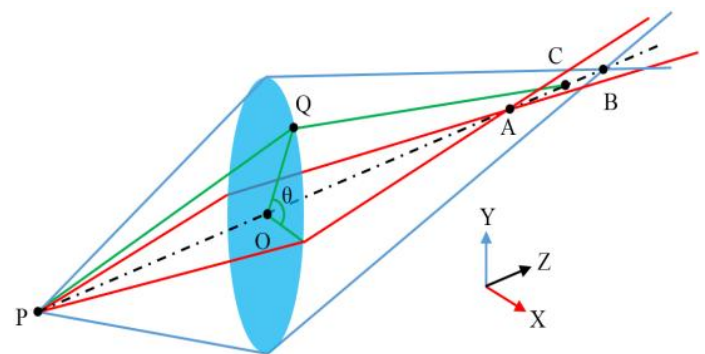

Fig. 5. The optical path of astigmatism.

In Fig. 5, point $\mathrm{P}$ represent a point on an object, point $\mathrm{O}$ is the center of the convex lens. The most curving meridian of the convex lens is called the primary meridian, its focus is point $\mathrm{A}$, and the meridian with minimum curvature is called the secondary meridian, its focus is point $\mathrm{B}$.

Rays emitted from point $\mathrm{P}$ and propagate in primary meridional plane will focus in point $\mathrm{A}$, and rays emitted from point $\mathrm{P}$ and propagate in secondary meridional plane will focus in point B. For those rays which neither propagates in primary meridional plane nor secondary meridional plane, such as point $\mathrm{Q}$ in Fig. 5, is assumed to pass through point $\mathrm{C}$. In this paper, the convex lens is considered axial symmetry in each meridional plane, as a result, point $\mathrm{C}$ will be in the optical axis, in other words, point $\mathrm{A}, \mathrm{B}$ and $\mathrm{C}$ are in the same line. The curvature of the meridian that point $\mathrm{Q}$ is on will be between the curvature of primary meridian and secondary meridian, it can be concluded that point $\mathrm{C}$ is between point $\mathrm{A}$ and point $\mathrm{B}$. In this paper, the distant between point $\mathrm{A}$ and point $\mathrm{C}$ is assumed to be proportional to the angle of the primary meridional plane and the meridional plane which point $\mathrm{Q}$ is in, which is $\theta$ shown in Fig. 5.

Compared with the simulation of myopia or the simulation of hyperopia, the simulation of astigmatism requires one more step: to determine the focal length $\mathrm{d}$. This focal length can be calculated by interpolating predefined max and min focal length according to the angle $\theta$.

\section{E. Glasses Prescription}

A typical glasses prescription may looks like:

$-2.00 \mathrm{DS} \quad-0.75 \mathrm{DC} \quad 160 \mathrm{~A}$

where DS means diopter sphere, a -2.00 diopter sphere lens's focal length is 0.5 meter. DC means diopter cylinder and A means axis. Minus DS represents myopia, plus DS means hyperopia. DC and A are for astigmatism.

From the point of view of human eye optical system simulation, the prescription can be used to calculate the focal length of the convex lens and the angle of the primary meridional plane.

Consider an eye with a diameter of $d_{\text {eye }}$ and need a $d s$ diopter sphere lens to correct, the focal length of the compound lenses, which consist of the convex lens of the eye and the glasses, is $d_{\text {eye }}$. The focal length of compound lenses of two thin lenses placed in contact is given by

$$
\frac{1}{f}=\frac{1}{f_{1}}+\frac{1}{f_{2}}
$$

Both the convex lens of the eye and the glasses can be regard as thin lens, and for contact lens, it is in contact with the eye.

In conclusion, the focal length of the convex lens of the eye is given by

$$
f=\frac{d_{\text {eye }}}{1-d_{\text {eye }} d s}
$$

As for the angle of the primary meridional plane, it is simply the value of $\mathrm{A}$ in degree under the prescription.

\section{F. Sampling}

For each pixel in the output image, all the rays that go through the convex lens and finally hit this pixel will make a contribution to the color of this pixel. Limited by the performance of computer, only some of the rays are simulated. These selected rays can be determined by the pixel point and the point in the convex lens that the ray goes through. The point in the convex lens is called sampling point. The distribution of these sampling points is the main topic disused in this section.

In the domain of ray tracing, sampling has a considerable impact on the output image. Random sampling and mean sampling are the easiest sampling methods, but it turns out that output is not quite satisfying.

Since the focus length in the simulation of astigmatism is influenced by the angle $\theta$ shown in Fig. 5, the more angles the sample points include, the better the output results will be. Therefore, a suitable sampling method for this simulation should as more angles as possible.

Another important aspect that must be taken account of is the distance between sampling points and the center. In order to avoid ghost effect, most of the sampling point should be around the center.

Considering all the factors mentioned above, the following sampling method is adopted (see Fig. 6).

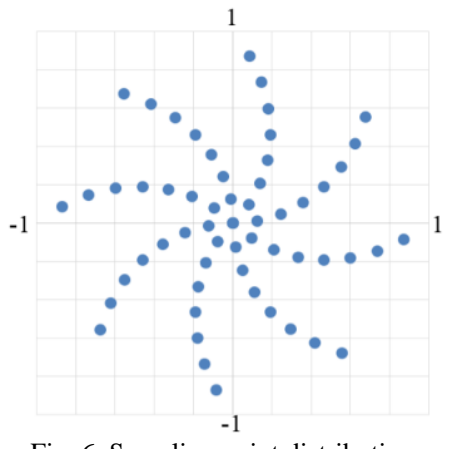

Fig. 6. Sampling point distribution 
The coordinate of the sampling point with index (DistantIndex, AngleIndex) can be calculated by the following formulas:

$$
\begin{aligned}
& X_{(\text {DistantIndex,Anglelndex })}=r \cos (\phi) \\
& Y_{(\text {Distantndex }, \text { Anglelndex })}=r \sin (\phi)
\end{aligned}
$$

where

$$
r=\text { DistantIndex / DistantFactor }
$$

$$
\phi=\frac{2 \pi}{\text { AngleFactor }}\left(\text { AngleIndex }+\frac{\text { DistantIndex }}{\text { DistantFactor }}\right)
$$

$$
\text { DistantIndex } \in[0, \text { DistantFactor })
$$

$$
\text { AngleIndex } \in[0, \text { AngleFactor })
$$

DistantFactor and AngleFactor are predefined constant integers.

\section{ENVIRONMENT LUMINANCE AND DEPTH OF FIELD}

People with myopia may have the experience that when the environment becomes brighter, the distant objects become clearer. This is because the pupil size will be adjusted in reaction to the bright environment, and thus changes the depth of field.

\section{A. Pixel Color and Environment Luminance}

The luminance of a single pixel located at $(x, y)$ is given by

$$
L(x, y)=0.3 R(x, y)+0.59 G(x, y)+0.11 B(x, y)
$$

where $R(x, y), G(x, y)$, and $B(x, y)$ are the red color, green color and blue color of the pixel, respectively.

Since the luminance of the pixels around the center should make a bigger contribution to the luminance of the whole image than those in the edge, Gaussian mixture model is adopted to combine the luminance of all the pixels in one image. The luminance of the environment $L_{e}$ is given by

$$
L_{e}=\sum\left[\frac{e^{-\left(a^{2}+b^{2}\right) / 2 \sigma^{2}}}{\operatorname{sum}} \mathrm{L}(x, y)\right]
$$

where

$$
\begin{gathered}
a=2 x / \text { ScreenWidth }-1 \\
b=2 y / \text { ScreenHeight }-1 \\
\text { sum }=\sum e^{-\left(a^{2}+b^{2}\right) / 2 \sigma^{2}}
\end{gathered}
$$

\section{B. Environment Luminance and Pupil Size}

According to the research of Blackie and Howland [13], the relationship of environment luminance and pupil size is given by

$$
D=5.697-0.658 \log L+0.007(\log L)^{2}
$$

where $D$ is the diameter of pupil, and $L$ is the luminance of environment.

\section{Pupil Size and Depth of Field}

The relationship between pupil size and depth of field can be illustrated by Fig. 7 and Fig. 8 .

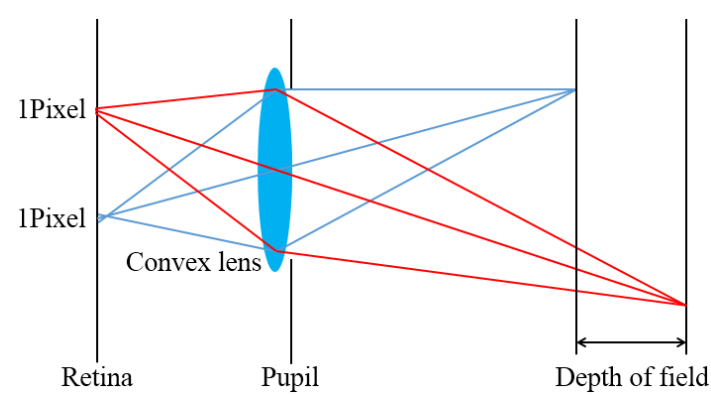

Fig. 7. Larger pupil, smaller depth of field

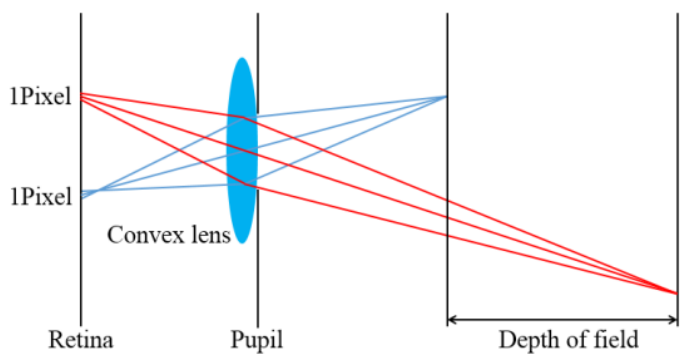

Fig. 8. Smaller pupil, larger depth of field

As vividly shown in Fig. 7 and Fig. 8, a larger pupil is accompanied by a smaller depth of field, and a smaller pupil results in smaller depth of field.

\section{RESULTS}

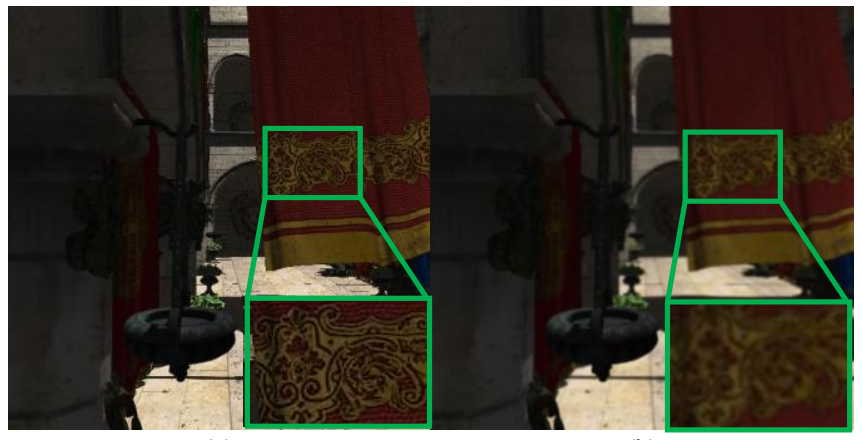

(b)

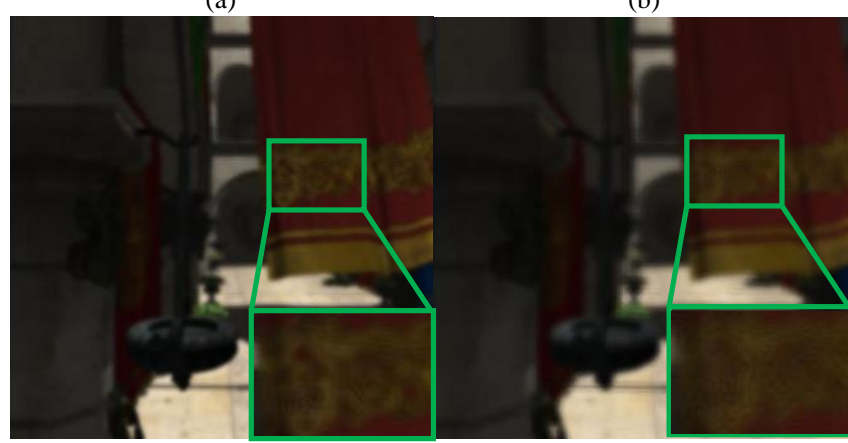

(c)

(d)

Fig. 9. Myopia result: (a) vision effect of normal eyes (b) vision effect of -1.5 DS myopia (c) vision effect of -3.0DS myopia (d) vision effect of -4.5 DS myopia.

In this section, render results of the simulation of myopia, hyperopia, astigmatism and depth of field variation will be 
presented.

The following images are rendered with both DistantFactor and AngleFactor set to 8, and the resolution of each image is $512 \times 512$.

In order to make the difference between the images easier to notice, part of each image will be enlarged.

\section{A. Myopia Results}

In Fig. 9, (a) is what normal eyes can see, and (b) (c) (d) are the vision effects of different degrees of myopia in which the distant objects are more and more blurry.

\section{B. Hyperopia Result}

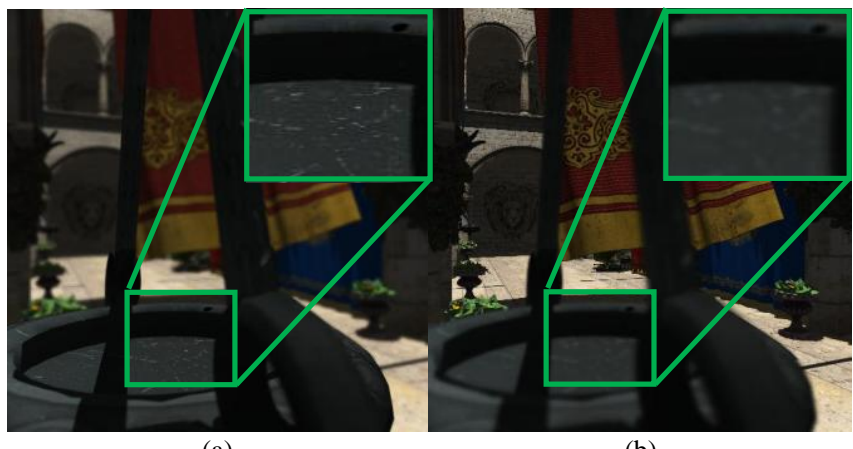

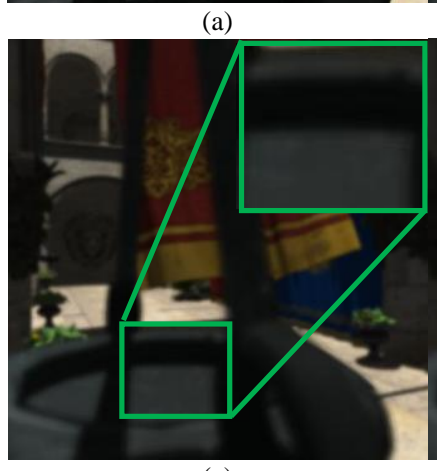

(c)

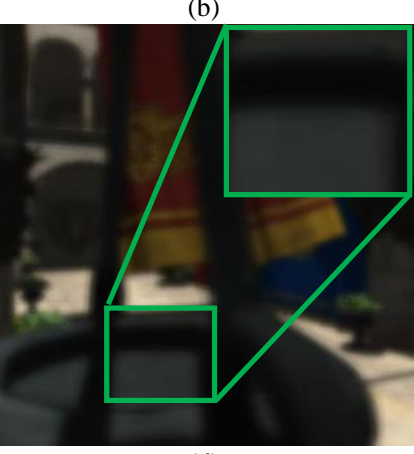

(d)
Fig. 10. Hyperopia result: (a) vision effect of normal eyes (b) vision effect of +2 .0DS hyperopia (c) vision effect of +4.0DS hyperopia (d) vision effect of +6.0DS hyperopia.

In Fig. 10, (a) is a normal eye staring at the near object. (b) (c) (d) are the visions of different degrees of hyperopia, which have difficulty focusing on the near objects.

\section{Astigmatism Result}

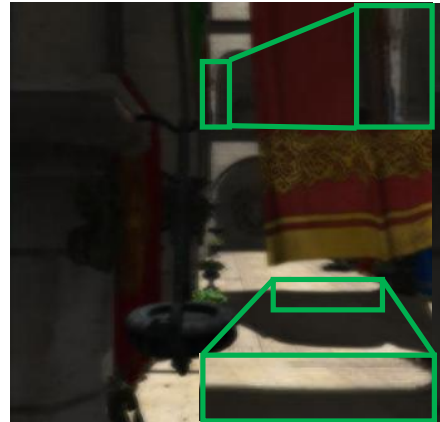

(a)

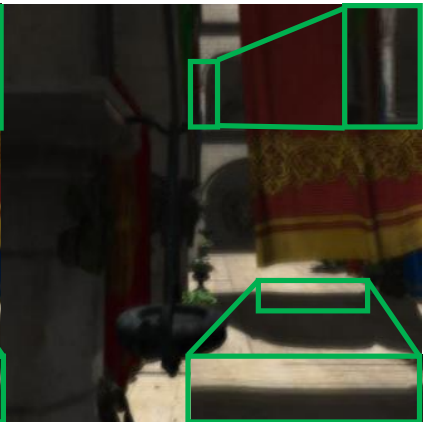

(b)
Fig. 11. Astigmatism result: (a) vision effect of -6.0DC 90A (b) vision effect of $-6.0 \mathrm{DC} 180 \mathrm{~A}$.

In Fig. 11, (a) is the vision of $-6.0 \mathrm{DC} 90 \mathrm{~A}$ and (b) is the vision of $-6.0 \mathrm{DC}$ 180A. The horizontal lines in (a) are relatively clearer than those in (b), while the vertical lines in (b) are relatively clearer than those in (a).

\section{Depth of Field Variation Result}

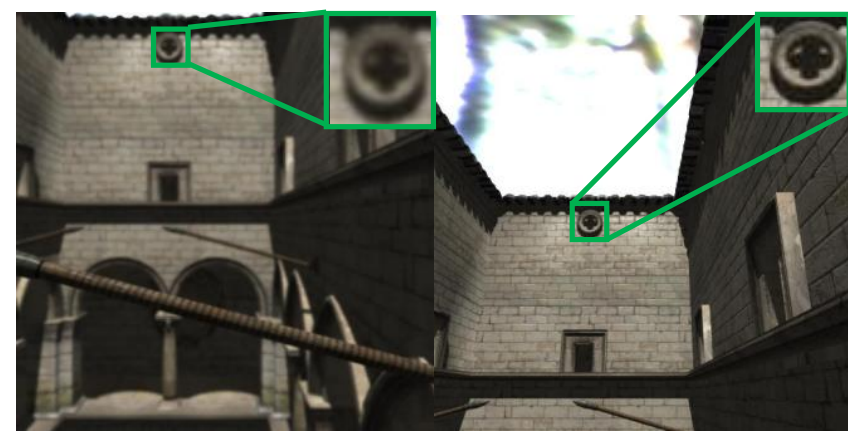

(a)

(b)

Fig. 12. Depth of field variation result: (a) vision effect of darker environment (b) vision effect of brighter environment.

In Fig. 12, (a) is relatively darker, the distant objects are blurred. (b) is the vision of the same parameters with brighter environment, and the same objects become clearer. The depth of field of the right image is larger than the left image.

\section{E. Sampling Methods Comparison}

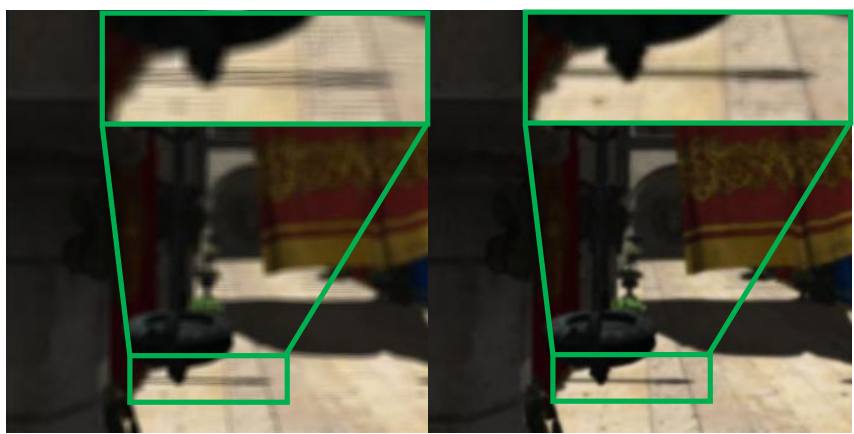

(a)

(b)

Fig. 13. Sampling methods comparison: (a) even sampling method (b) sampling method proposed in this paper.

In Fig. 13, (a) is rendered with a sampling method that the sampling points distribution is even distribution. (a) has ghost effect, while (b), which is render with the sampling method proposed in this paper, doesn't has any ghost effect and the blur is smooth.

\section{CONCLUSION}

In this paper, a simplified optical model is used to simulate the characteristics of human eye optical system, including myopia, hyperopia and astigmatism. In order to achieve realistic results, ray tracing is used throughout the simulation. But unlike the traditional ray tracing, the direction of rays when they are refracted by the eye is calculated with a novel and effective method. A suitable sampling method is proposed and makes significant contribution to the results. The variation of depth of field caused by the luminance of the environment is also simulated by adjusting the pupil size according to the brightness of current rendered image.

\section{ACKNOWLEDGMENT}

The authors wish to thank Arturo García, Francisco Ávila, Sergio Murguía and Leo Reyes for the ray tracing framework [16] and Crytek for the Sponza model. 


\section{REFERENCES}

[1] J. Angle and D. Wissmann, "The epidemiology of myopia," American Journal of Epidemiology, vol. 111, pp. 220-228, 1980

[2] D. A. Atchison and G. Smith, Optics of the Human Eye, 1st ed. 2000, pp. 5-6.

[3] K. Tsubota, B. Wachler, D. T. Azar, and D. D. Koch, Hyperopia and Presbyopia, 1st ed. Marcel Dekker, 2003, ch. 1.

[4] J. Porter, A. Guirao, I. G. Cox, and D. R. Williams, "Monochromatic aberrations of the human eye in a large population," JOSA A, vol. 18 , pp. 1793-1803, 2001.

[5] D. A. Goss and R. W. West, Introduction to the Optics of the Eye, 1st ed. Butterworth-Heinemann Boston, MA, 2001, ch. 3.

[6] S. De Groot and J. Gebhard, "Pupil size as determined by adapting luminance," JOSA, vol. 42, pp. 492-495, 1952.

[7] F. W. Campbell, "The depth of field of the human eye," Journal of Modern Optics, vol. 4, pp. 157-164, 1957.

[8] B. A. Barsky, A. W. Bargteil, D. D. Garcia, and S. A. Klein, "Introducing vision-realistic rendering," in Proc. Eurographics Rendering Workshop, 2002, pp. 26-28.

[9] B. A. Barsky, "Vision-realistic rendering: simulation of the scanned foveal image from wavefront data of human subjects," presented at the 1st Symposium on Applied perception in graphics and visualization, Los Angeles, California, 2004.

[10] W. Fink and D. Micol, "simEye: computer-based simulation of visual perception under various eye defects using Zernike polynomials,' Journal of biomedical optics, vol. 11, pp. 054011/1-054011/12, 2006.

[11] M. Potmesil and I. Chakravarty, "A lens and aperture camera model for synthetic image generation," ACM SIGGRAPH Computer Graphics, vol. 15, pp. 297-305, 1981.

[12] M. Pharr and G. Humphreys, Physically Based Rendering from Theory to Implementation, 2nd ed. Amsterdam; Boston Burlington, Mass.: Elsevier; Morgan Kaufmann, 2010, pp. 313-318.

[13] C. Blackie and H. Howland, "An extension of an accommodation and convergence model of emmetropization to include the effects of illumination intensity," Ophthalmic and Physiological Optics, vol. 19 pp. 112-125, 1999.

[14] B. Winn, D. Whitaker, D. Elliott, and N. J. Phillips, "Factors affecting light-adapted pupil size in normal human subjects," Investigative Ophthalmology \& Visual Science, vol. 35, pp. 1132-1137, 1994

[15] P. A. Stanley and A. K. Davies, "The effect of field of view size on steady- state pupil diameter," Ophthalmic and Physiological Optics, vol. 15 , pp. 601-603, 1995 .

[16] G. Arturo, Á. Francisco, M. Sergio, and R. Leo, "Interactive Ray Tracing Using the Compute Shader in DirectX 11," in GPU PRO 3, 1st ed. A K Peters/CRC Press, 2012, pp. 353-376.

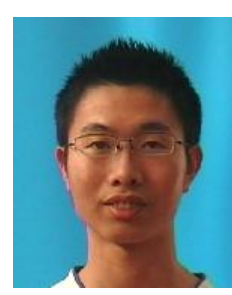

Zilong Wang was born in Guangdong, China in 1988. $\mathrm{He}$ received his bachelor engineer degree from the Shanghai Jiao Tong University in 2011. Since 2011, he has been a postgraduate student in Shanghai Jiao Tong University. His research interests include computer graphics and image processing.

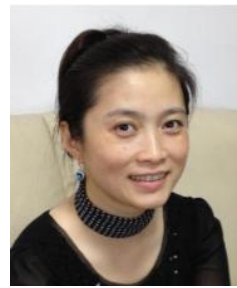

Shuangjiu Xiao was born in Sichuan Province in September 9, 1973. She received Ph.D. degree of Computer Aided Design in 2002 from Northwestern Polytechnical University, Xi'an, China. The major field of study of her includes computer graphics, human computer interaction.

She is an associate professor of School of Software of Shanghai Jiao Tong University. She did Postdoctor research during 2002-2004 in Computer Science and Technology Department of SJTU.

Prof. Xiao is a member of the China Graphics Society and the China Computer Federation. 